\title{
Aves como indicadoras ecológicas de etapas sucesionales en un bosque secundario, Antioquia, Colombia
}

\author{
Ángel David Salas Correa ${ }^{1} \&$ Néstor Javier Mancera-Rodríguez ${ }^{2 *}$
}

1. Universidad Nacional de Colombia, Sede Medellín, Postgrado en Bosques y Conservación Ambiental, Grupo Ecología y Conservación de Fauna Silvestre, Calle 59A No. 63-20, Medellín, Colombia; adsalasc@unal.edu.co

2. Universidad Nacional de Colombia, Sede Medellín. Departamento de Ciencias Forestales, Grupo Ecología y Conservación de Fauna Silvestre, Carrera 65 Nro. 59A - 110. Bloque 20 oficina 211, Medellín, Colombia; njmancer@unal.edu.co

* Correspondencia

Recibido 18-XII-2018. Corregido 31-VII-2019. Aceptado 20-XI-2019.

\begin{abstract}
Birds as ecological indicators of successional stages in a secondary forest, Antioquia, Colombia. Recovering areas from disturbance, after agriculture and livestock activities, increases secondary forests extension, which might change the quantity and quality of available habitat for birds. The Index of Biotic Integrity (IBI) compare the structure, composition and function of bird assemblages in secondary regeneration forest, from early succession to mature forest, to evaluate the ecological condition. Thus, the ecological condition along four stages of secondary forest in Antioquia, Colombia were studied using the IBI. This index was compared with richness and Shannon's diversity Index of the same bird assemblages. Five field surveys were conducted between June 2016 and February 2017, using fixed-radius point counts. In total 9516 individuals, from 187 bird species, belonging to 42 families and 15 orders were recorded. Habitat of occurrence, foraging guild and potential indicators groups were established for every species. Species richness and Punctual Abundance Index (PAI) of all categories were defined as candidate metrics, and after evaluating a total of 34 metrics, 13 were selected for further analysis. Metrics that were positively correlated with successional forest gradient, from highest to lowest disturbance, were: species richness of forest interior insectivorous, abundance of exclusively forest species, abundance of forest interior and edge species, abundance of endemic species and abundance of threatened species. Metrics that were negatively correlated with successional forest gradient, from highest to lowest disturbance, were: abundance of species from open areas, abundance of open areas and edge species, abundance of frugivorous, abundance of granivorous, abundance of omnivorous, abundance of insectivorous, abundance of scavengers and abundance of migratory species. IBI showed a positive significant linear correlation with the gradient of secondary forest regeneration, from early to mature forest, while Shannon's diversity Index and species richness showed a negative significant linear correlation. So that, in this particular case, IBI proved to be better indicator that the classical measures for assessing and interpreting ecological and environmental condition along the secondary forest succession evaluated.
\end{abstract}

Key words: foraging guild, multimetric indices, ecological indicators, tropical rainforest.

Salas Correa, A.D. \& Mancera-Rodríguez, N.J. (2020). Aves como indicadoras ecológicas de etapas sucesionales en un bosque secundario, Antioquia, Colombia. Revista de Biología Tropical, 68(1), 23-39.

En las últimas décadas, las actividades humanas como la agricultura y la ganadería han modificado drásticamente la composición, estructura y función de la mayor parte de los ecosistemas en los bosques del Neotrópico, deteriorando su calidad ecológica. Sin embargo, el abandono de estas actividades, y la recuperación de estas áreas para la restauración o 
regeneración natural permite que se presenten cambios en la estructura y composición de la vegetación con el avance de la sucesión de edades tempranas a maduras (Chazdon, 2014) y se generen cambios en la cantidad y calidad del hábitat que inciden en la organización de grupos taxonómicos y funcionales del ensamblaje de aves (Santamaría-Rivero, Leyequién, Hernández-Stefanoni, \& Wood, 2016).

Los índices de calidad biótica ayudan a evaluar y tomar medidas de los impactos que han alterado la integridad de los ecosistemas. En particular, el índice de integridad biótica (IIB), compara la estructura, composición y función de una comunidad biológica, de un ecosistema o una zona determinada, con el grado de disturbio (Karr, Fausch, Angermeier, Yant, \& Schlosser, 1986; O'Connell, Jackson, \& Brooks, 2000; Bryce, Hughes, \& Kaufmann, 2002; Glennon \& Porter, 2005; Bryce, 2006; Alexandrino et al., 2017). Este índice fue utilizado por primera vez para evaluar ecosistemas acuáticos para los ensamblajes de peces por Karr (1981) con base en múltiples métricas de las comunidades biológicas locales, su historia de vida y sus atributos ecológicos, comparando con sitios de referencia de mayor calidad ambiental (Karr et al., 1986; Karr \& Chu, 1999; Bryce, 2006).

El IIB ha sido modificado para evaluar ecosistemas terrestres, y a través del ensamblaje de aves y sus métricas biológicas se puede determinar la calidad de hábitat y la estructura de bosques en regeneración secundaria de una manera ágil y rápida (O'Connell et al., 2000; Bryce et al., 2002). El IIB basado en aves ha sido utilizado con éxito en bosques intervenidos y corredores ribereños (Bradford et al., 1998; O'Connell et al., 2000; Bryce et al., 2002; Glennon \& Porter, 2005; Bryce, 2006; Alexandrino et al., 2017) y adaptados a múltiples parámetros de la estructura del hábitat (Wilson \& Bayley, 2012).

Los ensamblajes de aves y sus grupos funcionales y taxonómicos, responden como indicadores biológicos, ya que muchas especies son sensibles a cambios ambientales, por lo que resultan apropiados para evaluar los cambios en la calidad y estructura de un ecosistema terrestre (O'Connell et al., 2000; Bryce et al., 2002; Sekercioglu et al., 2002; Chambers, 2008; Alexandrino et al., 2017). En este sentido, se entiende que los ambientes menos degradados mantendrían especies y gremios muy sensibles a la perturbación antrópica, mientras que los más alterados tendrían mayores abundancias de especies y gremios considerados poco sensibles a la perturbación humana o generalistas (O'Connell et al., 2000). Por lo tanto, las aves, pueden utilizarse para evaluar la calidad ecológica de fragmentos de bosque en proceso de regeneración secundaria, donde gradualmente aumenta la riqueza, abundancia, y composición de especies que cumplen importantes funciones en estos ecosistemas (Guariguata \& Ostertag, 2001; Chazdon, 2014; Letcher, et al., 2015; Norden, et al., 2015). El conocimiento existente y la comprensión de las comunidades de aves proporciona una guía para la selección de posibles métricas biológicas para evaluar la calidad ecológica de los fragmentos de bosque húmedo tropical (O'Connell et al., 2000; Bryce et al., 2002; Glennon \& Porter, 2005; Bryce, 2006).

En Colombia, los bosques siguen siendo degradados por la fragmentación de los ecosistemas, principalmente por actividades ganaderas y agrícolas y la expansión demográfica, determinando un paisaje dominante de fragmentos en forma de islas (Murcia, Kattan, \& Andrade-Pérez, 2013) y un mosaico de bosques en diferentes estados sucesionales. Por lo anterior, y teniendo en cuenta que los bosques tropicales secundarios desempeñan un importante papel en la conservación de la biodiversidad, este estudio tiene como objetivos: (i) evaluar la integridad ecológica a través del índice de integridad biótica (IIB) en un gradiente de disturbio antrópico, en cuatro diferentes estadios de sucesión secundaria en la Reserva Natural Hacienda San Pedro, ubicada en el Municipio Maceo, Departamento de Antioquia, Colombia y (ii) comparar la respuesta del IIB con los valores de los índices de Riqueza de especies e Índice de Shannon calculados en estos mismos cuatro estadios de sucesión secundaria. Se plantea como hipótesis de investigación 
que el IIB tendrá una correlación positiva con el gradiente de regeneración secundaria del bosque con menores valores en los estadios de sucesión temprana y mayores valores en los de sucesión avanzada, donde es menor la perturbación antrópica lo que permite la presencia de especies de aves más sensibles a la pérdida y deterioro de hábitat, indicando una mejor condición ecológica. A diferencia de los índices de Riqueza de especies e Índice de Shannon que destacan solamente el número de especies presentes y/o su relación con la abundancia de individuos en la comunidad, el IIB permite identificar métricas que evalúan rasgos funcionales de importancia para la comunidad y relacionarlas con el grado de disturbio para evaluar la condición ecológica de un ecosistema. Este estudio basado en el IIB de un ensamblaje de aves podría ser considerado como un modelo de evaluación de calidad ecológica para la conservación de áreas en proceso de regeneración natural o de restauración en los Andes colombianos y ser usado como referencia en zonas que han sufrido alto disturbio antrópico por actividades como la ganadería o la agricultura y que pueden ser destinadas a conservación.

\section{MATERIALES Y MÉTODOS}

Área de estudio: La Reserva Natural Hacienda San Pedro se encuentra ubicada en el noroccidente de la cordillera Central colombiana (6²7’35.0” N \& 74 47'15.0” W), cuenca media del Río Magdalena, en el municipio de Maceo, departamento de Antioquia, Colombia. Esta reserva se encuentra en zona de Bosque Húmedo Premontano (bh-PM) y Bosque Húmedo Tropical (bh-T), en un rango altitudinal 800 a 1100 m.s.n.m. (Salas-Correa \& Mancera-Rodríguez, 2018). La reserva tiene una extensión de 300 hectáreas, con predominio de bosque secundario en regeneración natural. Tiene una temperatura media de 23.8 ${ }^{\circ} \mathrm{C}$, una humedad relativa del $87 \%$ y una precipitación media anual de $2757 \mathrm{~mm}$ para la estación meteorológica Caracolí.

Para toda la reserva se evaluaron los cuatro estadios sucesionales de crecimiento del bosque secundario establecidos por SalasCorrea y Mancera-Rodríguez (2018): SS1: sucesión en edad de 0-5 años, con área de 30 ha., caracterizada por la presencia de pastos, escasa vegetación arbórea y donde anteriormente predominó la actividad ganadera; SS2: sucesión en edad de 10-13 años, con área de 50 ha., presenta vegetación arbórea, helechos y especies heliófilas de rápido crecimiento, anteriormente el área fue usada para actividades agrícolas; SS3: sucesión con edad de 18-20 años, con área de 40 ha., presenta algunos árboles de altura mayor a $30 \mathrm{~m}$., epífitas y especies tolerantes a la sombra, y anteriormente el área fue utilizada en actividades ganaderas; SS4: sucesión avanzada con edad mayor a 50 años, con área de 180 ha presenta grandes árboles, además de epifitas y bromelias, anteriormente el área fue sometida a extracción de árboles en forma selectiva.

Muestreo de aves: Se realizaron cinco muestreos de cuatro días consecutivos cada uno, entre junio 2016 y febrero 2017, con una frecuencia de muestreo bimestral. Para los cinco muestreos, en cada uno de los cuatro estadios sucesionales se establecieron cuatro puntos fijos de conteo de aves con radio de 15 m y separados $200 \mathrm{~m}$ uno de otro, ubicándolos al interior para evitar los efectos de borde entre sucesiones. En cada punto se registraron cuatro veces al día, durante 20 minutos, los registros visuales de las especies observadas y sus abundancias mediante la metodología de puntos fijos de conteo con el uso de binoculares entre las 6:00 a 10:00 h y las 14:00 a 18:00 h. El esfuerzo de muestreo total fue de 21.3 horas/ estadio/muestreo. Se identificaron las especies de aves en campo con las guías de Hilty y Brown (1986), Restall, Rodner y Lentino (2007) y la lista de especies se ordenó taxonómicamente de acuerdo al Comité de Clasificación de Suramérica -SACC (Remsen et al., 2018). La metodología más detallada, así como la relación entre la diversidad taxonómica y funcional del ensamblaje de aves y la estructura de vegetación en los cuatro estadios de 
sucesión secundaria estudiados se presentaron en Salas-Correa y Mancera-Rodríguez (2018).

Desarrollo del índice de integridad biótica basado en aves (IIB): El desarrollo de un IIB regional, depende de la identificación de un gradiente de perturbación humana y la recolección de datos biológicos para cada sitio (O’Connell, et al., 2000; Bryce et al., 2002). Para este estudio, el gradiente de perturbación fue dado por los cuatro estadios sucesionales de regeneración definidos por Salas-Correa y Mancera-Rodríguez (2018), en el que se relacionaron indicadores ambientales que representan la historia de degradación y crecimiento de cada estadio sucesional del bosque secundario estudiado como por ejemplo la edad media del bosque, y las variables estructurales de la vegetación en cada estadio como la altura promedio, la altura máxima, el área basal, el número árboles por hectárea, el número de especies y el número de especies por $\mathrm{m}^{2}$, para definir un intervalo de perturbación desde SS1 (mayor afectación por presencia de pastos, escasa vegetación arbórea y predominio de actividad ganadera) hasta el estadio SS4 (menor afectación con grandes árboles y dosel cubierto, y bosque de más de 50 años de regeneración natural) en el área estudiada. Por lo tanto, este rango representó un gradiente válido de disturbios para los puntos de conteo de aves.

Debido a que la reserva Hacienda San Pedro se encuentra en una zona altamente modificada por actividad ganadera y no existe un bosque cercano en condición de hábitat natural no intervenido, se optó por utilizar como referencia los mejores resultados que se obtuvieron de cada métrica, enfoque que ha sido utilizado ampliamente en otros estudios de IIB (e.g., Karr et al., 1986; Bryce et al., 2002; Bryce, 2006; Alexandrino et al., 2017).

Se identificaron las métricas del ensamblaje de aves, con base en los atributos de su historia natural que representan sus características funcionales, clasificando cada especie de acuerdo con tres componentes: (i) El hábitat de presencia, determinado por el hábitat en que cada especie fue observada: especies de interior de bosque (IB); especies asociadas a bordes o zonas de transición a áreas abiertas (BO); especies de áreas abiertas (AA), especies que puede aparecer en áreas abiertas y bordes (AA$\mathrm{BO})$; especies observadas en bordes e interior de bosque (BO-IB); y especies observadas en los tres hábitats (AA-BO-IB); (ii) los gremios de forrajeo, se definieron teniendo en cuenta la dieta primaria de cada especie de ave y se clasificó en: frugívoros (FR), granívoros (GR), nectarívoros (NE), carnívoros (CA), carroñeros (CR), omnívoros (OM), insectívoros (IN), siguiendo la propuesta de las guías de Hilty y Brown (1986) y Restall et al. (2007); y (iii) los grupos de indicadores potenciales, correspondieron a especies endémicas, que se definieron de acuerdo con Chaparro-Herrera, Echeverry-Galvis, Cordoba-Cordoba y Sua-Becerra (2013), especies migratorias, establecidas de acuerdo con Naranjo, Amaya, Eusse-González y Cifuentes-Sarmiento (2012), especies amenazadas, que se definieron mediante la categorización en riesgo de extinción de las listas rojas de la UICN, y especies insectívoras de interior de bosque (IN-IB), que son especies consideradas sensibles a la pérdida y fragmentación del hábitat (Sekercioglu et al., 2002; Sekercioglu, Daily, \& Ehrlich, 2004).

Para las seis categorías de hábitat de presencia, siete gremios de forrajeo y cuatro de grupos de indicadores potenciales, descritos anteriormente, se determinó la riqueza de especies y el Índice de abundancia puntual (IAP) (Bibby, Burgess, Hill, \& Mustoe, 2000; Vielliard, Almeida, Anjos, \& Silva, 2010) a partir del conteo de cada punto de muestreo, y éstos se usaron como las 34 métricas candidatas iniciales para el IIB (Tabla 1). Para determinar el IAP se tuvo en cuenta el número total de conteos visuales para cada especie en cada uno de los puntos de conteo, y se dividió por el número de muestreos hechos en cada punto, obteniendo un número no dimensional que permite comparaciones de especies entre sitios de muestra y/o períodos de tiempo (Vielliard et al., 2010; Alexandrino et al., 2017). La abundancia relativa de cada grupo funcional de aves (métricas candidatas) se obtuvo sumando el número 
TABLA 1

Selección de métricas de aves para su inclusión en el Índice de integridad biótica (IIB) de acuerdo a la metodología sugerida por Alexandrino et al. (2017)

TABLE 1

Selection of bird metrics for inclusion in the Index of Biotic Integrity (IBI) according to the methodology suggested by Alexandrino et al. (2017)

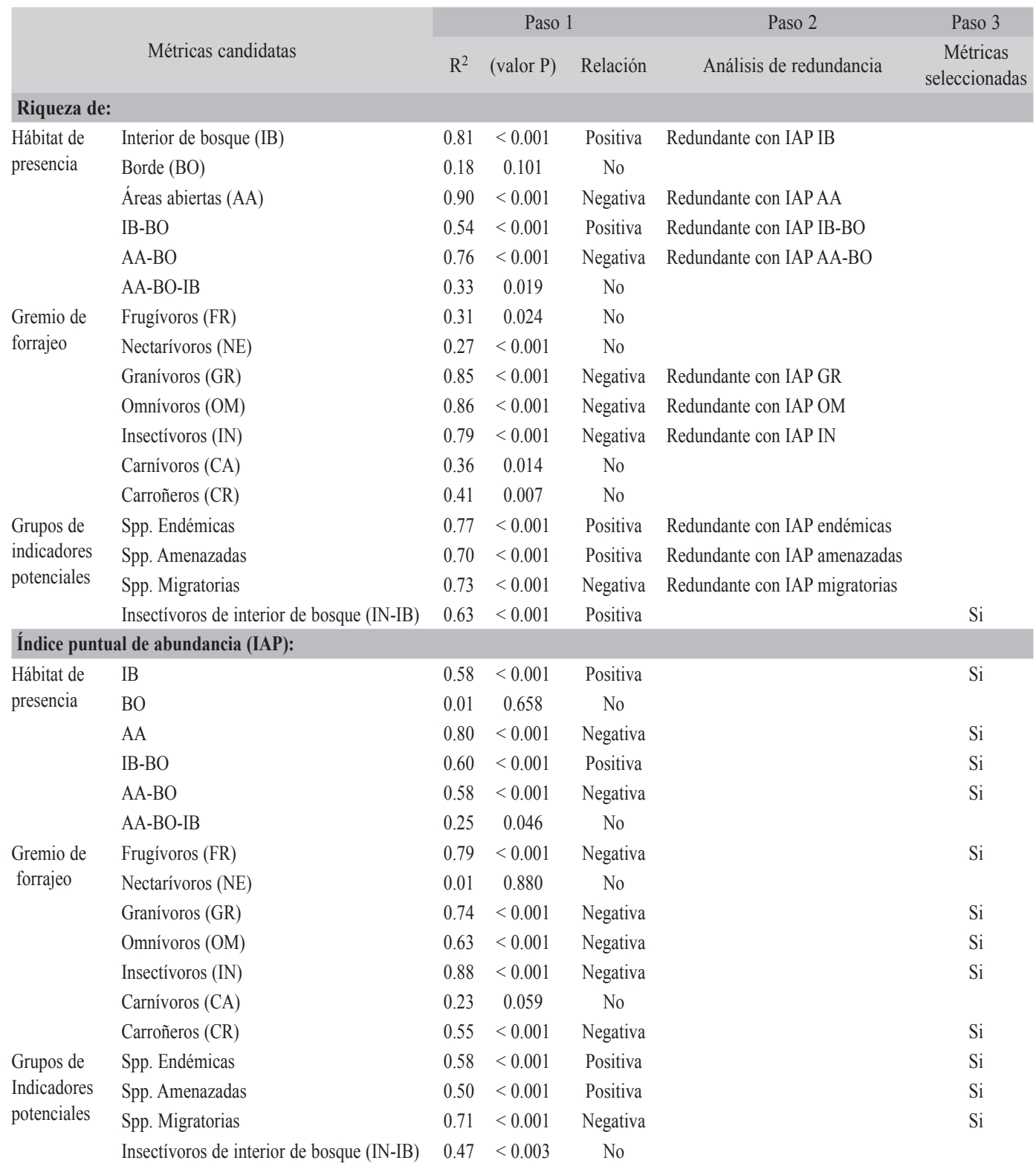

Paso 1: selección de 23 métricas candidatas que tenían una relación significativa con el rango de calidad de hábitat $\left(\mathrm{R}^{2}>\right.$ 0.5 y P < 0.001). Paso 2: identificación de métricas redundantes. Paso 3: selección de métricas para su inclusión en el IBI. Step 1: selection of 23 candidate metrics that had a significant relationship with the rank of habitat quality $\left(\mathrm{R}^{2}>0.5\right.$ and $\mathrm{P}$ $<0.001)$. Step 2: identification of redundant metrics. Step 3: selection of metrics for inclusion in the IBI 
de conteos visuales obtenido para cada especie que pertenece al grupo, y luego se calculó el IAP para el grupo respectivo.

De las 34 métricas candidatas iniciales del ensamblaje de aves, se seleccionaron 13 métricas finales, siguiendo la metodología de tres pasos propuesta por Alexandrino et al. (2017). En el paso 1 se seleccionaron 23 métricas candidatas que tenían una relación significativa con el gradiente de mayor a menor perturbación $\left(\mathrm{R}^{2}>0.5\right.$ y $\left.\mathrm{P}<0.001\right)$, en el paso 2 se identificaron las métricas redundantes y en el paso 3 se seleccionaron las 13 métricas finales para su inclusión en el IBI (Tabla 1).

Con las 13 métricas finales seleccionadas se construyó el IIB para cada uno de los cuatro puntos de muestreo de los 4 estadios en sucesión secundaria. Para cada métrica seleccionada se identificó su valor de referencia, que fue el valor más alto observado entre las puntuaciones. Luego, cada valor métrico observado en cada recuento de puntos se dividió con su valor de referencia y se multiplicó por 10 para obtener una escala de 0 (menor puntuación) a 10 (mayor puntuación). Para las métricas que tuvieron una respuesta negativa con el gradiente de perturbación humana, se hizo el puntaje inverso al restar 10 del puntaje obtenido para convertir el 10 en el peor puntaje y el 0 en el mejor. De acuerdo con Alexandrino et al. (2017) se calculó el IIB para cada conteo de puntos sumando cada métrica seleccionada ponderada (valores de 0 a 10) y dividiendo por los 13 indicadores válidos utilizados (e.g., Bryce et al., 2002; Bryce, 2006). Lo anterior, dio el resultado final para el IIB con un intervalo de 0-10 y esta escala se ajustó en intervalos de condición ecológica de acuerdo a la escala propuesta por Rodríguez-Olarte, Amaro, Coronel y Taphorn (2006): excelente $(>8)$, Bueno (6-8), regular (4-6), malo (2-4) y pobre $(<2)$.

Con el fin de comparar la respuesta del IIB con los valores de los índices de Riqueza de especies e Índice de Shannon (Magurran, 2004), calculados para cada punto de muestreo en estos mismos cuatro estadios de sucesión secundaria, se realizaron tres regresiones lineales independientes entre el IIB final, la riqueza total de especies y el índice de diversidad de Shannon como variables dependientes, y los cuatro estadios sucesionales, que reflejan el gradiente de las perturbaciones humanas, como la variable independiente, y se estimó el valor $\mathrm{R}^{2}$. Estos análisis se realizaron con el programa R Studio 3.5.2. (RStudio, 2017).

\section{RESULTADOS}

En total, se registraron 9516 individuos de 187 especies de aves pertenecientes a 42 familias y 15 órdenes y se definieron 34 diferentes métricas candidatas para evaluarlas en las cuatro etapas de la sucesión secundaria de bosque en la Reserva Hacienda San Pedro (Material Complementario) que se relacionaron con el gradiente de perturbaciones antrópicas, lo que permitió la selección de las 13 métricas finales para la construcción del IIB (Tabla 1).

De estas 13 métricas seleccionadas, cinco mostraron una relación positiva con el gradiente de perturbación de SS1 (mayor perturbación) a SS4 (menor perturbación y condición de referencia con bosque de más de 50 años de regeneración), mientras que ocho métricas mostraron una relación negativa (Tabla 1). Las métricas de integridad biótica que se relacionaron positivamente con el gradiente de mayor perturbación (SS1) a menor perturbación (SS4) fueron: riqueza de insectívoros de interior de bosque (Riqueza Insectívoros IN-IB: $\mathrm{R}^{2} 0.63$, $\mathrm{P}<0.001$ ), IAP de especies de Interior de Bosque (IAP IB: $\mathrm{R}^{2} 0.58, \mathrm{P}<0.001$ ), IAP de especies de interior y borde (IAP IB-BO: $\mathrm{R}^{2}$ $0.60, \mathrm{P}<0.001)$, IAP de especies endémicas (IAP Spp. endémicas: $\mathrm{R}^{2} 0.58, \mathrm{P}<0.001$ ), IAP de especies amenazadas (IAP Spp. Amenazadas: $\mathrm{R}^{2} 0.50, \mathrm{P}<0.001$ ) (Tabla 1 y Material Complementario).

Las métricas de integridad biótica que se relacionaron negativamente con el gradiente de mayor a menor perturbación fueron IAP de especies de áreas abiertas (IAP AA: $\mathrm{R}^{2} 0.80$, $\mathrm{P}<0.001$ ), IAP de especies de bordes y áreas abiertas (IAP AA-BO: $\mathrm{R}^{2} 0.58, \mathrm{P}<0.001$ ), IAP de especies del gremio frugívoros (IAP FR: $\mathrm{R}^{2}$ $0.79, \mathrm{P}<0.001)$, IAP de especies del gremio 
granívoros (IAP GR: $\mathrm{R}^{2} 0.74, \mathrm{P}<0.001$ ), IAP de especies del gremio omnívoros (IAP OM: $\left.\mathrm{R}^{2} 0.63, \mathrm{P}<0.001\right)$, IAP del gremio insectívoros (IAP IN: $\mathrm{R}^{2} 0.88, \mathrm{P}<0.001$ ), IAP de especies del gremio carroñeros (IAP CR: $\mathrm{R}^{2} 0.79$, $\mathrm{P}$ $<0.001$ ) y el IAP de especies migratorias (IAP Spp. Migratorias: $\mathrm{R}^{2} 0.71, \mathrm{P}<0.001$ ) (Tabla 1 y Material Complementario).

El IIB mostró que la integridad es mayor en los estadios en sucesión secundaria avanzada SS3 y SS4, donde es menor la perturbación antrópica, con un valor de IIB de 8.91 (D.E. = $0.55)$ y 8.20 (D.E. $=0.33$ ), respectivamente, reflejando una condición ecológica en categoría excelente. Mientras que el estadio SS2 presentó un valor de IIB de 4.68 (D.E. $=0.62$ ) con condición ecológica regular y el estadio SS1 un valor de IIB de 2.02 (D.E. $=0.86$ ) con condición ecológica mala. Se presentó una relación lineal positiva del IIB con el gradiente de mayor a menor perturbación $\left(\mathrm{R}^{2}=0.810\right.$, $\mathrm{P}<0.001)$, mientras que la riqueza total de especies $\left(\mathrm{R}^{2}=0.795, \mathrm{P}<0.001\right)$ y el índice de diversidad Shannon $\left(\mathrm{R}^{2}=0.652, \mathrm{P}<0.001\right)$ tuvieron una relación negativa con el gradiente de sucesión (Fig. 1), con mayores valores de riqueza total de especies y de índice de diversidad de Shannon en los estadios de sucesión iniciales SS2 y SS1.

\section{DISCUSIÓN}

Las métricas biológicas basadas en el IIB, evaluaron con efectividad la condición ecológica entre los diferentes estadios de sucesión y permitieron definir que los estadios SS3 y SS4 tienen una integridad biótica excelente, con especies de hábitos funcionales especializadas en nichos ecológicos puntuales, especies endémicas y especies en riesgo de extinción que requieren de bosques en mejor estado de conservación. Por su parte, los estadios iniciales SS2 y SS1 presentaron un hábitat atractivo para algunas especies de aves especialmente generalistas y los valores de IIB mostraron una condición ecológica regular a mala.

El IIB puede ser muy útil para evaluar la diversidad en un contexto más integral,
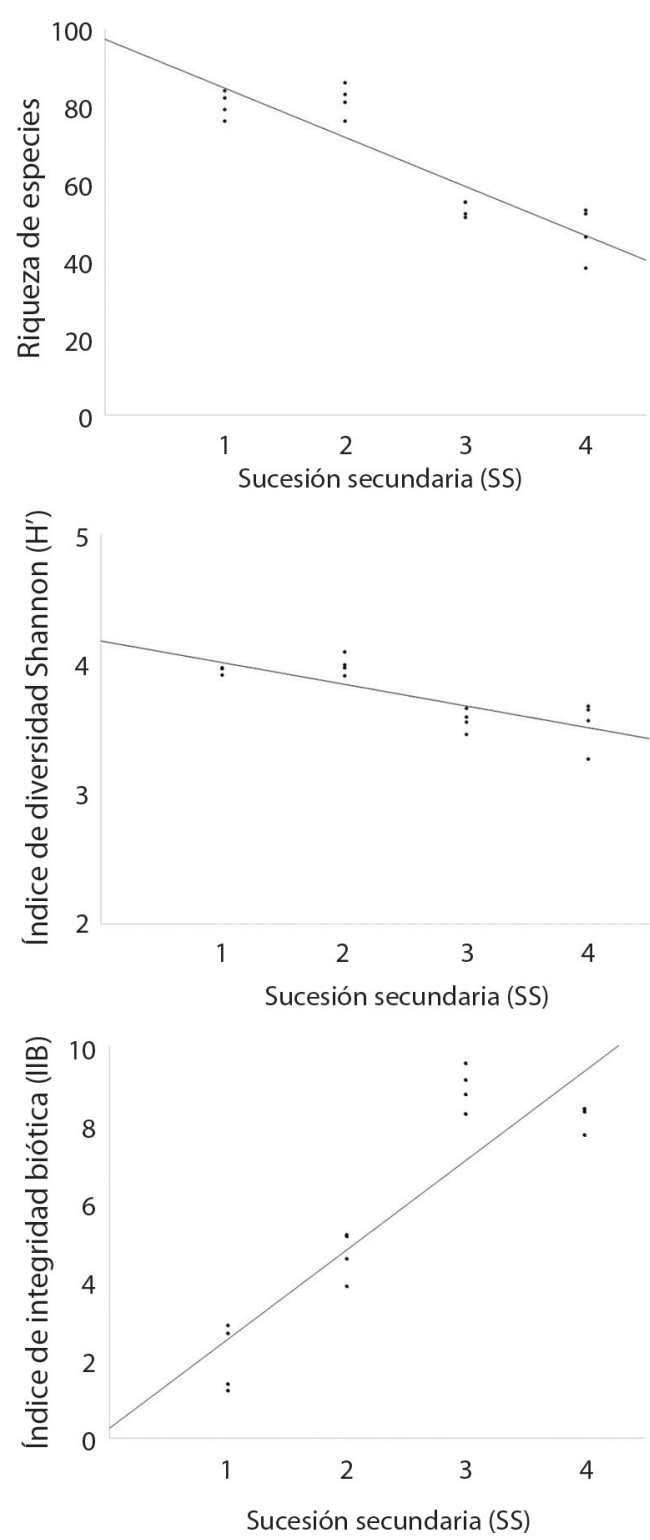

Fig. 1. Relación lineal entre la riqueza de especies, la diversidad de Shannon y el Índice de integridad biótica basado en aves (IIB) con el gradiente de sucesión secundaria del bosque húmedo tropical, en cuatro etapas de sucesión, desde edades tempranas (SS1) hasta maduras (SS4) en la Reserva Natural Hacienda San Pedro, Antioquia, Colombia. Fig. 1. Linear relationship between species richness, Shannon diversity, and our bird based Index of Biotic Integrity with the gradient of secondary rainforest succession, in four successional stages, from early (SS1) to mature ages (SS4) at Hacienda San Pedro Natural Reserve, Antioquia, Colombia. 
comparado con los índices de diversidad, ya que permitió establecer una relación entre la afectación del hábitat y la condición ecológica, siendo una herramienta de evaluación efectiva de la calidad de hábitat de estadios en regeneración que pueden definir áreas comparables a zonas bien conservadas, y puede ser usado como un indicador útil para la evaluación de bosques secundarios en regeneración. Así mismo, la utilización de métricas asociadas a los grupos funcionales facilitó la interpretación de condiciones ecológicas de cada estadio en sucesión, con lo cual se pueden tomar acciones de conservación en zonas concretas donde existe una mayor heterogeneidad de microhábitats, dado que una mayor diversidad de vegetación provee hábitats más específicos que solo pueden ser utilizados por especies particulares, como los estadios de sucesión SS3 y SS4.

Por su parte, los mayores valores de las medidas clásicas de riqueza de especies y el índice de diversidad de Shannon se encontraron en los estadios de sucesión iniciales SS2 y SS1. Esta mayor diversidad de especies de aves puede deberse a una mayor cantidad de ambientes y ecotonos que se presentan entre los estadios sucesionales iniciales e intermedios que permiten que a las especies generalistas que lograron permanecer allí durante el proceso de pérdida de hábitat, se sumen especies que empiezan a colonizar los nuevos ambientes generados por los procesos de regeneración natural donde se mezclan ambientes de áreas abiertas con bordes de bosques.

Este trabajo muestra que evaluar el ensamblaje de aves con las medidas clásicas de riqueza total de especies y el índice de diversidad de Shannon, no permite determinar efectivamente la condición ecológica de los distintos estadios sucesionales estudiados, ya que, aunque permiten conocer el número de especies presentes y/o su relación con la abundancia de individuos en la comunidad, estas no han sido diseñadas para medir como tal la condición ecológica. Sin embargo, se requiere ser cuidadoso con la interpretación de la respuesta taxonómica del ensamblaje de aves en los diferentes estados sucesionales ya que sólo se usaron estos dos índices de diversidad. Al respecto, Espejo y Morales (2019) encontraron que la diversidad taxonómica medida como riqueza basada en el número efectivo de especies con los valores de los números de Hill y el recambio y composición de especies de aves a partir de una matriz de similitud de abundancia de Bray-Curtis se correlacionó significativamente con la complejidad del hábitat, aumentando en estados sucesionales más complejos en diferentes estados de sucesión de bosque seco tropical en el sur del Valle del río Magdalena, en Colombia.

Los resultados del presente estudio constituyen un avance en el proceso de selección de las métricas para un ensamblaje de aves tropicales, con fines de encontrar grupos taxonómicos focales o gremios de forrajeo y grupos especializados de indicación en la evaluación de la condición ecológica en diferentes estadios de sucesión de bosque. Por ejemplo, especies insectívoras de interior de bosque (IN-IB) al igual que especies nectarívoras aumentaron su riqueza en los estadios SS3 y SS4 donde los valores de IIB fueron mayores. Estos estadios representan las sucesiones más avanzadas con una mejor condición ecológica del bosque en regeneración lo que, a lo largo del tiempo, favorece el desarrollo de nichos especializados para las aves en todos los estratos del bosque (Sekercioglu et al., 2002; Sekercioglu et al., 2004). La mejor condición ecológica en estos estadios está acompañada por mayor número de especies arbóreas y de altura media y máxima de la vegetación, y disminución de la disponibilidad de luz, el viento, la lluvia y las fluctuaciones de temperatura (Salas-Correa \& Mancera-Rodríguez, 2018) a diferencia de los estadios de sucesión inicial que se ven afectados por cambios mayores en los flujos físicos de radiación, viento y agua que pueden tener efectos importantes sobre la vegetación nativa y las interacciones bióticas (Saunders, Hobbs, \& Margules, 1991).

Las métricas de abundancia IAP de especie de aves de interior de bosque y de transición entre interior y bordes IB-BO también presentaron una relación positiva al gradiente de sucesión, de mayor a menor perturbación. 
Esta relación, se debe a la alta especificidad de hábitat que presentan las especies de interior de bosque y en particular gremios de forrajeo como IN-IB. Estas especies se relacionaron con una estructura del bosque caracterizada por tener árboles de mayor altura, mayor área basal, y una alta diversidad de especies vegetales (Salas-Correa \& Mancera-Rodríguez, 2018) que permiten, en última instancia, una mayor cantidad de nichos ecológicos específicos y mayor heterogeneidad ambiental en aquellos estadios sucesionales donde se mezclen ambientes de bordes con ambientes de bosques. Esto sugiere que la diversidad de especies especialistas aumenta con la sucesión y los cambios asociados con la estructura forestal (e.g. O'Connell et al., 2000; Leyequién, HernándezStefanoni, Santamaría-Rivero, Dupuy-Rada, \& Chable-Santos, 2014). En estadios SS2 y $\mathrm{SS} 3$, se presentan especies de aves de transición entre interior y bordes IB-BO capaces de adaptarse al empobrecimiento de los bosques maduros y su posibilidad de ocurrir en etapas tempranas de crecimiento secundario (Stotz, Fitzpatrick, Parker III, \& Moskovits, 1996; Imbeau, Drapeau, \& Mönkkönen, 2003; Uezu, Beyer, \& Metzger, 2008). Estas aves dominan etapas intermedias de regeneración y cumplen un rol ecológico importante como dispersores, polinizadores y controladores de insectos y otras especies.

Las otras métricas que presentaron relación positiva con el gradiente de perturbación de mayor a menor perturbación fueron IAP de especies endémicas y amenazadas. La presencia de tres especies endémicas Ortalis columbiana, Habia gutturalis y Capito hypoleucus, y de cinco especies categorizadas en riesgo de extinción (lista roja de la UICN) Odontophorus gujanensis, Habia gutturalis, Tinamus major Ramphastos vitellinus y Capito hypoleucus en el interior de las sucesiones más avanzadas (SS3 y SS4), demuestran que estas especies precisan de bosques en mejor estado de conservación para subsistir. Las especies endémicas y amenazadas proporcionan información sobre sitios prioritarios para su conservación (Bencke, Mauricio, Develey, \& Goerck, 2006) ya que son sensibles a las modificaciones del hábitat y su presencia puede ser una indicadora de alta integridad y calidad ambiental (e.g. Ribon, Simon, \& Mattos, 2003; Anjos, Bochio, Campos, McCrate, \& Palomino, 2009; Alexandrino et al., 2017). Así, estas especies tienen un valor adicional para la evaluación de la condición ecológica de los estadios de sucesión secundaria en bosques en restauración o regeneración natural.

Las métricas que tuvieron una relación negativa con el hábitat de referencia (e.g. IAP AA; IAP AA-BO; IAP OM; IAP IN) presentaron una alta relación con el gradiente de perturbación, siendo indicadoras de baja integridad biótica. Especies más generalistas (e.g. Crotophaga ani, Bubulcus ibis, Sporophila schistacea, Columbiana talpacoti, Milvago chimachima, Pitangus sulphuratus), fueron tolerantes a la perturbación antrópica, y se adaptaron a una estructura de vegetación con menores valores de área basal, altura máxima y menor número de árboles por hectárea asociadas a los estadios de sucesión SS1 y SS2 (Salas-Correa \& Mancera-Rodríguez, 2018). A nivel de gremios de forrajeo se observó que los IAP de los gremios OM, CR, GR, $\mathrm{CA}$ e IN tienen una relación negativa con el gradiente de perturbación de mayor a menor perturbación, presentando mayores valores en los estadios sucesionales iniciales. Los gremios OM (e.g. Bubulcus ibis, Phimosus infuscatus), CR (e.g. Cathartes aura, Coragyps atratus) y GR (e.g. Columbina talpacoti) se relacionaron con el estadio SS1, y los gremios CA (e.g. Elanoides forficatus, Milvago chimachima), e IN (e.g. Streptoprocne zonaris, Stelgidopteryx ruficollis) se asociaron con áreas de borde del estadio SS2. Aves de estos gremios utilizan bosques de menor integridad, tal vez debido al mayor hábitat de borde disponible y el acceso al hábitat circundante ya que buscan su alimento preferentemente en áreas abiertas (O'Connell et al., 2000).

La métrica basada en la abundancia relativa de aves migratorias respondió también negativamente al gradiente de mayor a menor perturbación, siendo más abundantes en áreas 
con mayor intervención antrópica de las sucesiones SS1 y SS2. Al respecto, Naranjo et al. (2012) mencionan que los hábitats usados por especies migratorias, están siendo altamente intervenidos por actividades humanas y algunas especies como rapaces migratorias o paseriformes, dependen de zonas boscosas para buscar alimento o descansar y son afectadas por la deforestación y la consecuente destrucción de sus hábitats. Esto posiblemente indica que muchas especies de aves migratorias tienen un alto nivel de tolerancia a hábitats modificados y su amplia distribución, su movilidad y su capacidad de obtención de recursos, les permiten utilizar una gran gama de hábitats (e.g. Cerezo, Robbins, \& Dowell, 2009; Naranjo et al., 2012; Díaz-Bohórquez, Bayly, Botero, \& Gómez, 2014).

Declaración de ética: los autores declaran que todos están de acuerdo con esta publicación y que han hecho aportes que justifican su autoría; que no hay conflicto de interés de ningún tipo; y que han cumplido con todos los requisitos y procedimientos éticos y legales pertinentes. Todas las fuentes de financiamiento se detallan plena y claramente en la sección de agradecimientos. El respectivo documento legal firmado se encuentra en los archivos de la revista.

\section{AGRADECIMIENTOS}

A los propietarios de la Reserva Natural Hacienda San Pedro. Al convenio de becas Globo Común del SENESCYT, al Instituto Ecuatoriano de Crédito Estudiantil (IECE) y al Instituto Colombiano de Crédito Educativo y Estudios Técnicos en el Exterior - ICETEX (Colombia).

\section{RESUMEN}

Las áreas que se recuperan perturbaciones tales como actividades agrícolas y ganaderas, generan un aumento de bosques secundarios que causan cambios en la cantidad y calidad del hábitat disponible para las aves. El Índice de Integridad Biótica (IIB) compara la estructura, composición y función del ensamblaje de las aves en una regeneración secundaria del bosque, desde edades tempranas hasta maduras, para evaluar su condición ecológica. Se estudió la integridad ecológica utilizando el IIB en cuatro etapas de sucesión de bosque secundario. El IBI se calculó utilizando ensamblajes de aves encontrados en estas cuatro etapas de la sucesión de bosques, y su desempeño se comparó con la riqueza general y el índice de diversidad de Shannon. Se realizaron cinco muestreos de campo entre junio 2016 y febrero 2017, en los que se registraron las aves utilizando recuentos de puntos de radio fijo. En total, se registraron 9516 individuos de 187 especies de aves pertenecientes a 42 familias y 15 órdenes. Para cada especie se estableció hábitat de presencia, gremio de forrajeo y grupos de indicadores potenciales. La riqueza de especies y el Índice de Abundancia Puntual (PAI) de todas las categorías, se utilizaron como métricas candidatas y luego de evaluar un total de 34 métricas, se seleccionaron 13 para los análisis. Las métricas que se correlacionaron positivamente con el gradiente, de mayor a menor perturbación, fueron: riqueza de insectívoros de interior de bosque, abundancia de especies exclusivamente de interior de bosque, abundancia de especies de interior y borde, abundancia de especies endémicas y abundancia de especies amenazadas. Las métricas que se correlacionaron negativamente con el gradiente, de mayor a menor perturbación, fueron abundancia de especies de áreas abiertas, abundancia de especies de áreas abiertas y de borde, abundancia de frugívoros, abundancia de granívoros, abundancia de omnívoros, abundancia de insectívoros, abundancia de carroñeros y abundancia de especies migratorias. El IBI mostró una correlación lineal significativa positiva con el gradiente de regeneración secundaria del bosque, desde edades tempranas hasta maduras, mientras que la riqueza total de especies y el índice de diversidad de Shannon mostraron una correlación lineal negativa. El IBI, en este caso particular, demostró ser mejor que las medidas clásicas de la riqueza de especies y el índice de diversidad de Shannon para evaluar e interpretar la condición ecológica y ambiental de las etapas de la sucesión del bosque secundario evaluado.

Palabras clave: gremios de forrajeo, índices multimétricos, indicadores ecológicos, bosque húmedo tropical.

\section{REFERENCIAS}

Alexandrino, E. R., Buechley, E. R., Karr, J. R., de Barros, K. M. P. M., de Barros Ferraz, S. F., do Couto, H. T. Z., \& Şekercioglu, C. H. (2017). Bird based Index of Biotic Integrity: Assessing the ecological condition of Atlantic Forest patches in human-modified landscape. Ecological Indicators, 73, 662-675.

Anjos, L. D., Bochio, G. M., Campos, J. V., McCrate, G. B., \& Palomino, F. (2009). Sobre o uso de níveis de sensibilidade de aves à fragmentação florestal na avaliação da Integridade Biótica: um estudo de caso 
no norte do Estado do Paraná, sul do Brasil. Revista Brasileira de Ornitologia, 17(1), 28-36.

Bencke, G. A., Mauricio, G. N., Develey, P. F., \& Goerck, J. M. (2006). Áreas importantes para a Conservação das aves no Brasil: parte 1, estados do domínio da Mata Atlântica. São Paulo, Brasil: SAVE.

Bibby, C. J., Burgess, N. D., Hill, D. A., \& Mustoe, S. H. (2000). Bird Census Techniques ( $2^{\text {nd }}$ ed.). San Diego, Academic Press Limited.

Bradford, D. F., Franson, S. E., Neale, A. C., Heggem, D. T., Miller, G. R., \& Canterbury, G. E., (1998). Bird species assemblages as indicators of biological integrity in GreatBasin rangeland. Environmental Monitoring and Assessment, 49, 1-22.

Bryce, S. A. (2006). Development of a bird integrity index: measuring avian response to disturbance in the Blue Mountains of Oregon, USA. Environmental Management, 38(3), 470-486.

Bryce, S. A., Hughes, R. M., \& Kaufmann, P. R. (2002). Development of a bird integrity index: using bird assemblages as indicators of riparian condition. Environmental Management, 30(2), 294-310.

Cerezo, A., Robbins, C. S., \& Dowell, B. (2009). Uso de hábitats modificados por aves dependientes de bosque tropical en la región caribeña de Guatemala. Revista de Biología Tropical, 57, 401-419.

Chambers, S. A. (2008). Birds as environmental indicators: review of literature. Melbourne: Parks Victoria.

Chaparro-Herrera, S., Echeverry-Galvis, M. A., CórdobaCórdoba, S., \& Sua-Becerra, A. (2013). Listado actualizado de las aves endémicas y casi-endémicas de Colombia. Biota Colombiana, 14(2), 235-272.

Chazdon, R. (2014). Second growth: the promise of tropical forest regeneration in an age of deforestation. Chicago, U.S.A.: University of Chicago Press.

Díaz-Bohórquez, A. M., Bayly, N. J., Botero, J. E., \& Gómez, C. (2014). Aves migratorias en agroecosistemas del norte de Latinoamérica, con énfasis en Colombia. Ornitología Colombiana, 14, 3-27.

Espejo, N., \& Morales, N. (2019). Variación de la diversidad taxonómica y funcional de la avifauna en un bosque seco tropical (bs-T) en diferentes estados de sucesión en el sur del Valle del Magdalena, Huila, Colombia. Caldasia, 41(1), 108-123.

Glennon, M. J., \& Porter, W. F. (2005). Effects of land use management on biotic integrity: an investigation of bird communities. Biological Conservation, 126(4), 499-511.

Guariguata, M., \& Ostertag, R. (2001). Neotropical secondary forest succession: changes in structural and functional characteristics. Forest Ecology and Management, 148, 185-206.

Hilty, S., \& Brown, B. (1986). A guide to the birds of Colombia. New Jersey: Princeton University Press.

Imbeau, L., Drapeau, P., \& Mönkkönen, M. (2003). Are forest birds categorized as "edge species" strictly associated with edges? Ecography, 26(4), 514-520.

Karr, J. R. (1981). Assessment of biotic integrity using fish communities. Fisheries, 6(6), 21-27.

Karr, J. R., \& Chu, E. W. (1999). Restoring life in running waters: Better biological monitoring. Covelo, California: Island Press.

Karr, J. R., Fausch, K. D., Angermeier, P. L., Yant, P. R., \& Schlosser, I. J. (1986). Assessment of biological integrity in running water: a method and its rationale (Special Publication 5). Champaign, Illinois: Illinois Natural History Survey.

Letcher, S., Lasky, J., Chazdon, R., Norden, N., Wright, S., Meave, J., \& Andrade, J. (2015). Environmental gradients and the evolution of successional habitat specialization: a test case with 14 Neotropical forest sites. Journal of Ecology, 103(5), 1276-1290.

Leyequién, E., Hernández-Stefanoni, J., Santamaría-Rivero, W., Dupuy-Rada, J., \& Chable-Santos, J. (2014). Effects of tropical successional forests on bird feeding guilds. In N. Nakagoshi \& J. A. Mabuhay (Eds.), Designing Low Carbon Societies in Landscapes, Ecological Research Monographs (pp. 177-202). Tokyo: Springer.

Magurran, A. E. (2004). Measuring Biological Diversity. Oxford: Blackwell.

Murcia, C., Kattan, G., \& Andrade-Pérez, G. (2013). Conserving Biodiversity in a Complex Biological and Social Setting: The Case of Colombia. In S. N. Sodhi, L. Gibson, \& P. H. Raven. Conservation Biology: Voices from the Tropics ( $1^{\text {st }}$ ed., pp.86-96). New Jersey: John Wiley \& Sons, Ltd. Published.

Naranjo, L., Amaya, J., Eusse-González, D., \& CifuentesSarmiento, Y. (2012). Guía de las Especies Migratorias de la Biodiversidad en Colombia. Aves (Vol. 1). Bogotá, D.C. Colombia: Ministerio de Ambiente y Desarrollo Sostenible/WWF Colombia

Norden, N., Angarita, H., Bongers, F., Martínez-Ramos, M., Granzow-de la Cerda, I., Van Breugel, M., \& Finegan, B. (2015). Successional dynamics in Neotropical forests are as uncertain as they are predictable. Proceedings of the National Academy of Sciences, 112(26), 8013-8018.

O’Connell, T. J., Jackson, L. E., \& Brooks, R. P. (2000). Bird guilds as indicators of ecological condition in the central Appalachians. Ecological Applications, 10(6), 1706-1721. 
Remsen, J. V., Areta, J. I., Cadena, C. D., Claramunt, S., Jaramillo, A., Pacheco, J. F., ... Zimmer, K. J. (2018). A classification of the bird species of South America. American Ornithologists' Union. Recuperado de http://www.museum.lsu.edu/ Remsen/SACCBaseline.html

Restall, R., Rodner, C., \& Lentino, M. (2007). Birds of Northern South America: An Identification Guide (Volume 1 \& 2). U.S.A.: Yale University Press.

Ribon, R., Simon, J. E., \& Mattos, G. T. (2003). Bird extinctions in Atlantic forest fragments of the Vicosa region: southeastern Brazil. Conservation Biology, $17,1827-1839$.

Rodríguez-Olarte, D., Amaro, A., Coronel, J., \& Taphorn, D. (2006). Integrity of fluvial fish communities is subject to environmental gradients in mountain streams, Sierra de Aroa, north Caribbean coast, Venezuela. Neotropical Ichthyology, 4(3), 319-328.

RStudio (2017). RStudio: Integrated development environment for R. Boston, Massachusetts, U.S.A.

Salas-Correa, A. D., \& Mancera-Rodríguez, N. J. (2018). Relaciones entre la diversidad de aves y la estructura de vegetación en cuatro etapas sucesionales de bosque secundario, Antioquia, Colombia. Revista U.D.C.A Actualidad \& Divulgación Científica, 21(2), 519-529.

Santamaría-Rivero, W., Leyequién, E., Hernández-Stefanoni, J., \& Wood, P. (2016). Influence of landscape structure and forest age on the richness and abundance of different bird feeding guilds and forestdependent birds in a seasonal dry tropical forest of Yucatan, Mexico. Tropical Ecology, 57(2), 313-332.
Saunders, D. A., Hobbs, R. J., \& Margules, C. R. (1991). Biological consequences of ecosystem fragmentation: a review. Conservation Biology, 5, 18-32.

Sekercioglu, C. H., Daily, G. C., \& Ehrlich, P. R. (2004). Ecosystem consequences of bird declines. Proceedings of the National Academy of Sciences USA, 101, 18042-18047.

Sekercioglu, C. H., Ehrlich, P. R., Daily, G. C., Aygen, D., Goehring, D., \& Sandi, R. F. (2002). Disappearance of insectivorous birds from tropical forest fragments. Proceedings of the National Academy of Sciences USA, 99(1), 263-267.

Stotz, D. F., Fitzpatrick, J. W., Parker III, T. A., \& Moskovits, D. K. (1996). Neotropical birds: ecology and conservation. U.S.A.: University of Chicago Press.

Uezu, A., Beyer, D. D., \& Metzger, J. P. (2008). Can agroforest woodlots work as stepping stones for birds in the Atlantic Forest region? Biodiversity and Conservation, 17(8), 1-16.

Vielliard, J. M. E., Almeida, M. E. C., Anjos, L., \& Silva, W. R. (2010). Levantamento quantitativo por pontos de escruta e o indice pontual de abundância (IPA). In S. V. Matter, F. C. Straube, I. Accordi, V. Piacentini, \& J. F. Cândido-Jr (Eds.), Ornitologia e Conservação. Ciência Aplicada, Técnicas de Pesquisa e Levantamento (pp. 47-60). Rio de Janeiro: Technical Books.

Wilson, M. J., \& Bayley, S. E. (2012). Use of single versus multiple biotic communities as indicators of biological integrity in northern prairie wetlands. Ecological Indicators, 20, 187-195. 


\section{MATERIAL COMPLEMENTARIO}

Especies registradas y sus características ecológicas en cuatro etapas de sucesión en la Reserva Natural Hacienda San Pedro, Antioquia, Colombia (Esta información se utilizó para identificar las 34 métricas candidatas)

\section{SUPPLEMENTARY MATERIAL}

Species recorded and their ecological characteristics in four successional stages at Hacienda San Pedro Natural Reserve, Antioquia, Colombia (This information was used to identify the 34 candidate metrics)

\begin{tabular}{|c|c|c|c|c|c|}
\hline Orden & Familia & Especie & $\begin{array}{l}\text { Hábitat de } \\
\text { presencia }\end{array}$ & $\begin{array}{l}\text { Gremio de } \\
\text { forrajeo }\end{array}$ & $\begin{array}{l}\text { Estado de } \\
\text { amenaza }\end{array}$ \\
\hline \multirow[t]{2}{*}{ Tinamiformes } & Tinamidae & Crypturellus soui & BO-IB & FR & \\
\hline & & Tinamus major & IB & FR & NT \\
\hline \multirow[t]{6}{*}{ Pelecaniformes } & Ardeidae & Ardea alba* & AA & $\mathrm{OM}$ & \\
\hline & & Bubulcus Ibis* & AA & $\mathrm{OM}$ & \\
\hline & & Butorides striata & AA-BO & $\mathrm{OM}$ & \\
\hline & & Egretta thula* & AA & $\mathrm{OM}$ & \\
\hline & & Tigrisoma fasciatum & IB & CR & \\
\hline & Threskiornithidae & Phimosus infuscatus & AA & $\mathrm{OM}$ & \\
\hline \multirow{5}{*}{ Accipitriformes } & Cathartidae & Sarcoramphus papa & AA & $\mathrm{CR}$ & \\
\hline & & Cathartes aura* & AA & $\mathrm{CR}$ & \\
\hline & & Coragyps atratus & AA & $\mathrm{CR}$ & \\
\hline & Accipitridae & Elanoides forficatus* & BO & CA & \\
\hline & & Rupornis magnirostris & AA-BO & CA & \\
\hline \multirow[t]{3}{*}{ Falconiformes } & Falconidae & Caracara cheriway & AA & $\mathrm{CA}$ & \\
\hline & & Herpetotheres cachinnans & BO & $\mathrm{CA}$ & \\
\hline & & Milvago chimachima & AA-BO & $\mathrm{CA}$ & \\
\hline \multirow[t]{2}{*}{ Galliformes } & Cracidae & Ortalis columbiana** & BO-IB & FR & \\
\hline & Odontophoridae & Odontophorus gujanensis & IB & FR & NT \\
\hline Gruiformes & Rallidae & Aramides cajanea & AA & $\mathrm{OM}$ & \\
\hline \multirow[t]{4}{*}{ Columbiformes } & Columbidae & Columbina talpacoti & AA & GR & \\
\hline & & Leptotila verreauxi & BO-IB & FR & \\
\hline & & Patagioenas cayennensis & AA & FR & \\
\hline & & Patagioenas subvinacea* & AA & FR & VU \\
\hline \multirow[t]{5}{*}{ Psittaciformes } & Psittacidae & Ara ararauna & IB & FR & \\
\hline & & Brotogeris jugularis & $\mathrm{AA}$ & FR & \\
\hline & & Eupsittula pertinax & BO-IB & FR & \\
\hline & & Forpus conspicillatus & AA & FR & \\
\hline & & Pionus menstruus & BO-IB & FR & \\
\hline \multirow[t]{2}{*}{ Cuculiformes } & Cuculidae & Crotophaga ani & AA & IN & \\
\hline & & Piaya cayana & BO & IN & \\
\hline \multirow[t]{5}{*}{ Apodiformes } & Apodidae & Streptoprocne zonaris & AA & IN & \\
\hline & Trochilidae & Amazilia amabilis & $\mathrm{BO}$ & $\mathrm{NE}$ & \\
\hline & & Amazilia tzacatl & AA-BO & $\mathrm{NE}$ & \\
\hline & & Anthracothorax nigricollis & AA-BO & NE & \\
\hline & & Chalybura buffonii & AA-BO & $\mathrm{NE}$ & \\
\hline
\end{tabular}


MATERIAL COMPLEMENTARIO / SUPPLEMENTARY MATERIAL

\begin{tabular}{|c|c|c|c|c|c|}
\hline Orden & Familia & Especie & $\begin{array}{l}\text { Hábitat de } \\
\text { presencia }\end{array}$ & $\begin{array}{c}\text { Gremio de } \\
\text { forrajeo }\end{array}$ & $\begin{array}{c}\text { Estado de } \\
\text { amenaza }\end{array}$ \\
\hline \multirow{47}{*}{ Passeriformes } & \multirow{13}{*}{ Cardinalidae } & Damophila julie & AA-BO & $\mathrm{NE}$ & \multirow{47}{*}{ NT } \\
\hline & & Florisuga mellivora & AA-BO & NE & \\
\hline & & Glaucis hirsutus & BO-IB & NE & \\
\hline & & Heliothryx barroti & $\mathrm{BO}$ & NE & \\
\hline & & Phaethornis anthophilus & BO-IB & $\mathrm{NE}$ & \\
\hline & & Phaethornis longirostris & BO-IB & $\mathrm{NE}$ & \\
\hline & & Phaethornis striigularis & $\mathrm{BO}$ & NE & \\
\hline & & Phaethornis syrmatophorus & BO-IB & NE & \\
\hline & & Habia gutturalis** & IB & IN & \\
\hline & & Pheucticus ludovicianus* & AA-BO & IN & \\
\hline & & Piranga rubra* & BO-IB & IN & \\
\hline & & Saltator coerulescens & AA & GR & \\
\hline & & Saltator maximus & AA & GR & \\
\hline & Coerebidae & Coereba flaveola & AA-BO & NE & \\
\hline & Corvidae & Cyanocorax affinis & AA-BO & $\mathrm{OM}$ & \\
\hline & Cotingidae & Lipaugus fuscocinereus & IB & FR & \\
\hline & \multirow[t]{2}{*}{ Emberizidae } & Arremon aurantiirostris & BO-IB & IN & \\
\hline & & Chlorospingus canigularis & BO-IB & FR & \\
\hline & Formicariidae & Formicarius analis & IB & IN & \\
\hline & \multirow[t]{3}{*}{ Fringillidae } & Euphonia laniirostris & AA-BO & FR & \\
\hline & & Euphonia música & AA-BO & FR & \\
\hline & & Euphonia xanthogaster & $\mathrm{BO}$ & FR & \\
\hline & \multirow[t]{11}{*}{ Furnariidae } & Dendrocincla fuliginosa & IB & IN & \\
\hline & & Dendroplex picus* & $\mathrm{BO}$ & IN & \\
\hline & & Glyphorynchus spirurus & IB & IN & \\
\hline & & Lepidocolaptes souleyetii & AA-BO & IN & \\
\hline & & Philydor fuscipennis & IB & IN & \\
\hline & & Sittasomus griseicapillus & BO-IB & IN & \\
\hline & & Synallaxis albescens & IB & IN & \\
\hline & & Xenops minutus & IB & IN & \\
\hline & & Xiphorhynchus guttatus & IB & IN & \\
\hline & & Xiphorhynchus picus & IB & IN & \\
\hline & & Xiphorhynchus susurrans & IB & IN & \\
\hline & \multirow[t]{2}{*}{ Hirundinidae } & Notiochelidon cyanoleuca & AA & IN & \\
\hline & & Stelgidopteryx ruficollis & AA & IN & \\
\hline & \multirow[t]{7}{*}{ Icteridae } & Agelaius icterocephalus & AA & GR & \\
\hline & & Icterus auricapillus & AA & IN & \\
\hline & & Icterus icterus & AA & IN & \\
\hline & & Molothrus bonariensis & AA & IN & \\
\hline & & Molothrus oryzivorus & AA & IN & \\
\hline & & Psarocolius decumanus & AA-BO-IB & FR & \\
\hline & & Psarocolius wagleri & AA-BO & IN & \\
\hline & \multirow{5}{*}{$\begin{array}{l}\text { Mimidae } \\
\text { Parulidae }\end{array}$} & Mimus gilvus & AA & OM & \\
\hline & & Basileuterus rufifrons & BO-IB & IN & \\
\hline & & Cardellina canadensis* & IB & IN & \\
\hline & & Dendroica castanea* & $\mathrm{BO}$ & IN & \\
\hline & & Dendroica fusca* & BO-IB & IN & \\
\hline
\end{tabular}


MATERIAL COMPLEMENTARIO / SUPPLEMENTARY MATERIAL

\begin{tabular}{|c|c|c|c|c|c|}
\hline Orden & Familia & Especie & $\begin{array}{r}\text { Hábitat de } \\
\text { presencia }\end{array}$ & $\begin{array}{l}\text { Gremio de } \\
\text { forrajeo }\end{array}$ & $\begin{array}{c}\text { Estado de } \\
\text { amenaza }\end{array}$ \\
\hline & \multirow{13}{*}{ Pipridae } & Leiothlypis peregrina* & $\mathrm{BO}$ & IN & \\
\hline & & Leiothlypis ruficapilla* & $\mathrm{BO}$ & IN & \\
\hline & & Mniotilta varia* & $\mathrm{BO}$ & IN & \\
\hline & & Myiothlypis fulvicauda & AA-BO-IB & IN & \\
\hline & & Oporornis philadelphia* & $\mathrm{BO}$ & IN & \\
\hline & & Oreothlypis peregrina* & $\mathrm{BO}$ & IN & \\
\hline & & Phaeothlypis fulvicauda & $\mathrm{BO}$ & IN & \\
\hline & & Setophaga castanea* & BO-IB & IN & \\
\hline & & Setophaga fusca* & BO-IB & IN & \\
\hline & & Setophaga ruticilla* & BO-IB & IN & \\
\hline & & Wilsonia canadensis* & IB & IN & \\
\hline & & Machaeropterus regulus & IB & FR & \\
\hline & & Manacus manacus & IB & FR & \\
\hline & Polioptilidae & Polioptila plumbea & AA & IN & \\
\hline & Thamnophilidae & Myrmotherula pacifica & BO & IN & \\
\hline & \multirow{32}{*}{ Thraupidae } & Thamnophilus atrinucha & IB & $\mathrm{IN}$ & \\
\hline & & Thamnophilus doliatus & BO-IB & $\mathrm{IN}$ & \\
\hline & & Chlorophanes spiza & $\mathrm{BO}$ & FR & \\
\hline & & Dacnis cayana* & $\mathrm{BO}$ & IN & \\
\hline & & Dacnis lineata & BO & FR & \\
\hline & & Eucometis penicillata & BO-IB & IN & \\
\hline & & Hemispingus superciliaris & IB & IN & \\
\hline & & Hemispingus verticalis & IB & IN & \\
\hline & & Hemithraupis flavicollis & IB & IN & \\
\hline & & Heterospingus xanthopygius & IB & FR & \\
\hline & & Oryzoborus angolensis & AA & GR & \\
\hline & & Oryzoborus crassirostris & AA & GR & \\
\hline & & Oryzoborus nuttingi & AA & GR & \\
\hline & & Ramphocelus dimidiatus & AA & FR & \\
\hline & & Ramphocelus flammigerus & AA-BO & FR & \\
\hline & & Ramphocelus icteronotus & AA & FR & \\
\hline & & Sicalis flaveola & AA & GR & \\
\hline & & Sporophila crassirostris & AA & GR & \\
\hline & & Sporophila minuta & AA & GR & \\
\hline & & Sporophila nigricollis & AA & GR & \\
\hline & & Sporophila schistacea & AA & GR & \\
\hline & & Tachyphonus luctuosus & AA-BO & FR & \\
\hline & & Tachyphonus rufus & AA-BO & FR & \\
\hline & & Tangara cyanicollis & AA & FR & \\
\hline & & Tangara girola & IB & FR & \\
\hline & & Tangara inornata & AA & FR & \\
\hline & & Tangara larvata* & AA & FR & \\
\hline & & Tangara vassorii & AA & FR & \\
\hline & & Tersina viridis & AA & FR & \\
\hline & & Thraupis cyanocephala & B & FR & \\
\hline & & Thraupis episcopus & AA & FR & \\
\hline & & Thraupis palmarum & AA & FR & \\
\hline
\end{tabular}


MATERIAL COMPLEMENTARIO / SUPPLEMENTARY MATERIAL

\begin{tabular}{|c|c|c|c|c|c|}
\hline Orden & Familia & Especie & $\begin{array}{l}\text { Hábitat de } \\
\text { presencia }\end{array}$ & $\begin{array}{l}\text { Gremio de } \\
\text { forrajeo }\end{array}$ & $\begin{array}{c}\text { Estado de } \\
\text { amenaza }\end{array}$ \\
\hline & \multirow{3}{*}{ Tityridae } & Pachyramphus cinnamomeus & AA-BO & $\mathrm{IN}$ & \\
\hline & & Pachyramphus rufus & AA & $\mathrm{IN}$ & \\
\hline & & Tityra semifasciata & BO-IB & $\mathrm{IN}$ & \\
\hline & \multirow[t]{5}{*}{ Troglodytidae } & Campylorhynchus griseus & AA & $\mathrm{IN}$ & \\
\hline & & Henicorhina leucosticta & IB & IN & \\
\hline & & Microcerculus marginatus & IB & $\mathrm{IN}$ & \\
\hline & & Pheugopedius fasciatoventris & IB & $\mathrm{IN}$ & \\
\hline & & Troglodytes aedon & AA & IN & \\
\hline & \multirow[t]{3}{*}{ Turdidae } & Catharus minimus* & IB & $\mathrm{IN}$ & \\
\hline & & Catharus ustulatus* & IB & FR & \\
\hline & & Turdus ignobilis & AA & FR & \\
\hline & \multirow[t]{28}{*}{ Tyrannidae } & Camptostoma obsoletum & BO-IB & IN & \\
\hline & & Colonia colonus & AA & IN & \\
\hline & & Contopus virens* & $\mathrm{BO}$ & IN & \\
\hline & & Elaenia flavogaster & AA & FR & \\
\hline & & Fluvicola pica & IB & IN & \\
\hline & & Legatus leucophaius & AA-BO & IN & \\
\hline & & Leptopogon amaurocephalus & $\mathrm{BO}$ & IN & \\
\hline & & Leptopogon superciliaris & BO-IB & $\mathrm{IN}$ & \\
\hline & & Machetornis rixosa & $\mathrm{AA}$ & $\mathrm{IN}$ & \\
\hline & & Megarynchus pitangua & BO & IN & \\
\hline & & Mionectes oleagineus & BO-IB & FR & \\
\hline & & Myiarchus cephalotes & AA-BO & IN & \\
\hline & & Myiarchus tuberculifer* & $\mathrm{BO}$ & $\mathrm{IN}$ & \\
\hline & & Myiodynastes luteiventris* & $\mathrm{BO}$ & IN & \\
\hline & & Myiodynastes maculatus* & AA-BO & $\mathrm{IN}$ & \\
\hline & & Myiopagis viridicata & AA & IN & \\
\hline & & Myiozetetes cayanensis & AA & IN & \\
\hline & & Myiozetetes similis & AA & IN & \\
\hline & & Oncostoma olivaceum & $\mathrm{BO}$ & IN & \\
\hline & & Pitangus sulphuratus & AA & $\mathrm{OM}$ & \\
\hline & & Pyrocephalus rubinus & AA & IN & \\
\hline & & Sayornis nigricans & AA-BO & IN & \\
\hline & & Serpophaga cinérea & AA & IN & \\
\hline & & Todirostrum cinereum & AA-BO & IN & \\
\hline & & Tolmomyias assimilis & IB & IN & \\
\hline & & Tyrannus melancholicus* & AA & IN & \\
\hline & & Tyrannus savana* & AA & IN & \\
\hline & & Zimmerius chrysops & BO-IB & IN & \\
\hline & Vireonidae & Hylophilus flavipes & AA & IN & \\
\hline Podicipediformes & Podicipedidae & Tachybaptus dominicus & $\mathrm{BO}$ & FR & \\
\hline Charadriiformes & Charadriidae & Vanellus chilensis* & AA & IN & \\
\hline \multirow[t]{3}{*}{ Coraciiformes } & \multirow[t]{2}{*}{ Alcedinidae } & Chloroceryle americana & $\mathrm{BO}$ & $\mathrm{CA}$ & \\
\hline & & Megaceryle torquata & $\mathrm{BO}$ & $\mathrm{CA}$ & \\
\hline & Momotidae & Baryphthengus martii & IB & $\mathrm{OM}$ & \\
\hline \multirow[t]{2}{*}{ Piciformes } & Capitonidae & Capito hypoleucus** & IB & FR & EN \\
\hline & Picidae & Celeus loricatus & IB & $\mathrm{IN}$ & \\
\hline
\end{tabular}


MATERIAL COMPLEMENTARIO / SUPPLEMENTARY MATERIAL

\begin{tabular}{|c|c|c|c|c|c|}
\hline Orden & Familia & Especie & $\begin{array}{l}\text { Hábitat de } \\
\text { presencia }\end{array}$ & $\begin{array}{c}\text { Gremio de } \\
\text { forrajeo }\end{array}$ & $\begin{array}{c}\text { Estado de } \\
\text { amenaza }\end{array}$ \\
\hline & \multirow{11}{*}{ Ramphastidae } & Colaptes punctigula & AA & IN & \\
\hline & & Dryocopus lineatus & AA-BO & IN & \\
\hline & & Melanerpes formicivorus & AA-BO & FR & \\
\hline & & Melanerpes pucherani & AA-BO & FR & \\
\hline & & Melanerpes rubricapillus & AA & $\mathrm{IN}$ & \\
\hline & & Picumus olivaceus & AA-BO & $\mathrm{IN}$ & \\
\hline & & Veniliornis dignus & $\mathrm{BO}$ & $\mathrm{IN}$ & \\
\hline & & Veniliornis kirkii & IB & $\mathrm{IN}$ & \\
\hline & & Pteroglossus torquatus & $\mathrm{BO}$ & FR & \\
\hline & & Ramphastos swainsonii & BO-IB & FR & \\
\hline & & Ramphastos vitellinus & BO-IB & FR & VU \\
\hline
\end{tabular}

Especie Migratoria (*), Especie Endémica (**). Casi Amenazada (NT). Vulnerable (VU). En Peligro (EN). Hábitat de presencia: áreas abiertas (AA), interior de bosque (IB) y bordes (BO). Gremios tróficos: frugívoros (FR), insectívoros (IN), granívoros (GR), nectarívoros (NE), omnívoros (OM), carnívoros (CA), carroñeros (CR).

Migratory species $\left(^{*}\right)$, Endemic species $(* *)$. Near Threatened (NT). Vulnerable (VU) Endangered (EN). Habitat of occurrence: open areas (AA), forest interior (IB) and edges (BO). Foraging guild: frugivorous (FR), insectivorous (IN), granivorous (GR), nectarivorous (NE), omnivorous (OM), carnivorous (CA), scavengers $(\mathrm{CR})$. 